\title{
Osteoporosis secundarias
}

\section{Secondary osteoporosis}

\section{J. Lafita ${ }^{1}$, J. Pineda ${ }^{2}$, C. Fuentes ${ }^{2}$, J. P. Martínez ${ }^{2}$}

\section{RESUMEN}

Se denomina osteoporosis secundaria a aquella que es causada por patologías o medicaciones, distintas a la pérdida ósea explicable por la etapa postmenopáusica o envejecimiento. Las posibles patologías que pueden condicionar la pérdida de masa ósea son muy variadas: endocrinológicas, digestivas, genéticas, hematológicas, reumáticas, post-transplante, farmacológicas y un amplio grupo misceláneo. En el artículo se revisan esencialmente las causas endocrinológicas, con especial énfasis en los aspectos más controvertidos en la actualidad, seguidos de una aproximación clínica para el diagnóstico sistemático de estas patologías, frecuentes en los casos etiquetados inicialmente de osteoporosis primaria.

Palabras clave. Osteoporosis secundaria. Estados hipogonadales. Patología tiroidea. Hiperparatiroidismo.

\begin{abstract}
Secondary osteoporosis is caused by pathologies or medications, differing from the bone loss explainable by the post-menopausal stage or by ageing. The possible pathologies that can condition the loss of bone mass are very varied: endocrinological, digestive, genetic, haematological, rheumatic, post-transplant, pharmacological and a wide miscellaneous group. This article essentially reviews the endocrinological causes, with special emphasis on the more controversial aspects, followed by a clinical approach for the systematic diagnosis of these pathologies, which are frequent in cases initially labelled as primary osteoporosis.
\end{abstract}

Key words. Secondary Osteoporosis. Hypogonadal states. Thyroid pathology. Hyperarathyroidism

An. Sist. Sanit. Navar. 2003; 26 (Supl. 3): 53-62.

1. Médico Adjunto de Endocrinología. Centro de Consultas Externas Príncipe de Viana. Pamplona.

2. Médico Residente de Endocrinología. Centro de Consultas Externas Príncipe de Viana. Pamplona.

\section{Correspondencia:}

Javier Lafita Tejedor

Servicio de Endocrinología

Centro de Consultas Externas Príncipe de Viana

C/ Irunlarrea, 3

31008 Pamplona

Tfno. 848-422038 


\section{OSTEOPOROSIS SECUNDARIAS}

Desde la segunda o tercera década de la vida, época en la que se consigue el pico máximo de masa ósea, se produce una pérdida progresiva más o menos marcada según la época del ciclo vital, que va a condicionar que, en determinados casos, se superen los límites establecidos de descenso de masa ósea (T-score $\leq-2,5$ ), llegándose al diagnóstico de osteoporosis ${ }^{1}$. Se utiliza el término de osteoporosis primaria para aquellas situaciones en que la disminución de masa ósea, puede ser explicada por los cambios involutivos del envejecimiento, así como por los cambios hormonales de la menopausia; reservando el concepto de osteoporosis secundaria a aquella que es causada o exacerbada por otras patologías o medicaciones ${ }^{2}$. La prevalencia de casos de osteoporosis secundaria es muy variable, en dependencia de la edad, sexo, grupo racial, etc. a que nos refiramos. Además, no siempre podemos hablar de una causa aislada, como origen de muchos casos de osteoporosis, sino que es relativamente frecuente que nos encontremos ante un conjunto de ellas imbricadas. Así la prevalencia de casos de osteoporosis secundaria, si nos circunscribimos a varones diagnosticados de osteoporosis, es hasta del $64 \%^{3}$. En mujeres perimenopausicas la prevalencia está próxima al $50 \%$, disminuyendo después de la menopausia hasta un nivel no desdeñable del 20 al $30 \%{ }^{2}$.

Las causas de osteoporosis secundarias son múltiples (Tabla 1): enfermedades genéticas, endocrinas, gastrointestinales, hematológicas, reumatológicas, nutricionales, farmacológicas, etc. Este amplio abanico de patologías, algunas con muy poca expresividad clínica, va a exigir la realización de estudios protocolizados y orientados clínicamente, que deben escalonarse de acuerdo a su rentabilidad diagnóstica, ajustándonos a la mayor eficacia, con un coste razonable.

Dado que el comentario pormenorizado de todas estas patologías supera ampliamente los objetivos del presente capítulo, vamos a centrarnos en aquellas que tienen más interés desde el punto de vista endocrino-metabólico, bien por su
Tabla 1. Causas de osteoporosis secundaria.

1- Enfermedades endocrinológicas

A- Estados hipogonadales

Insensibilidad a los andrógenos

Trastornos de la conducta alimentaria

Amenorrea de las atletas

Hiperprolactinemia

Panhipopituitarismo

Menopausia precoz

Síndromes de Turner o Klinefelter

B- Patologías endocrinas

Acromegalia

Insuficiencia suprarrenal

Enfermedad de Cushing

Diabetes mellitus tipo 1

Hiperparatiroidismo primario y

secundario

Secreción tumoral de PTH related peptide

Hipertiroidismo

Déficit nutricionales (Calcio, vitamina D, Magnesio, etc)

2- Enfermedades gastrointestinales

Enfermedad celiaca

Gastrectomía

Malabsorcion

Enfermedad inflamatoria intestinal

Cirrosis biliar primaria

Enfermedad hepática grave

Insuficiencia pancreática exocrina

3- Trastornos genéticos

Hemocromatosis

Hipofosfatasia

Osteogénesis imperfecta

Síndrome de Ehlers-Danlos

Síndrome de Marfan

Síndrome de Menkes

Síndrome de Riley-Day

Porfirias

Enfermedades de depósito

4- Trastornos hematológicos

Mieloma múltiple

Leucemias y linfomas

Mastocitosis sistémica

Anemia perniciosa

5- Enfermedades reumáticas

Artritis reumatoide

Espondilitis anquilosante

6- Transplante de órganos

Transplante de médula

Transplante de riñón, hígado, corazón o pulmón

7- Drogas

Anticoagulantes: heparina y cumarínicos

Anticomiciales

Ciclosporina y Tacrolimus

Drogas citotóxicas

Glucocorticoides y ACTH

Agonistas de la hormona liberadora de gonadotropinas

Metotrexate 
8- Miscelánea

Alcoholismo

Amiloidosis

Sarcoidosis

Fibrosis quística

Insuficiencia cardíaca congestiva

Enfisema

Enfermedad renal terminal

Hipercalciuria idiopática

Esclerosis múltiple

Nutrición parenteral

frecuencia o porque recientemente haya habido aportaciones importantes, en cuanto a su fisiopatología, diagnóstico o manejo; con excepción hecha de la osteoporosis asociada al hipercortisolismo o tratamiento con corticoides, que por su frecuencia e importancia ha requerido un capítulo individual en esta monografía.

\section{OSTEOPOROSIS ASOCIADA A ESTADOS HIPOGONADALES}

La repercusión esquelética del déficit de estrógenos o andrógenos va a ser muy diferente, en dependencia de la época de la vida en que se desarrolle; así los casos de hipogonadismo primario o secundario, en el período de adquisición de masa ósea, van a condicionar un menor pico de masa ósea y, sin embargo, si el déficit es posterior, se producirá un incremento de la actividad osteoclástica, con mediación de citokinas y una pérdida de masa ósea superior a la observada en población con estatus hormonal normal ${ }^{4}$.

Ejemplos claros de hipogonadismo primario los encontramos en los síndromes de Turner o de Klinefelter, el primero con fenotipo femenino y el segundo masculino.

El síndrome de Turner se caracteriza por un déficit parcial o total en la síntesis de estrógenos, secundario a la existencia de una monosomía total o en mosaico del par de cromosomas sexuales (XO), o bien a delecciones o recombinaciones del cromosoma $X$, que conllevan la presencia de una disgenesia gonadal. La incidencia de síndrome de Turner se aproxima a 1 cada 2.500 nacidos vivos, con una expresividad fenotípica muy variable: pacientes con talla baja, amenorrea en el $90 \%$ de los casos, con diversas malformaciones cardiacas, esqueléticas, renales, etc ${ }^{5}$. Las alteraciones esqueléticas: relación anómala segmento superior / segmento inferior y moderada osteopenia, incluso antes del período de desarrollo puberal, sugiere un cierto tipo de displasia ósea en estas pacientes. Una vez que ha comenzado la pubertad se aprecia una clara disminución de densidad mineral ósea, respecto a sus coetáneas, del 19 al 27\%, reducción similar a la apreciada en otros casos de amenorrea primaria, lo que sugiere que el déficit de estrógenos es la causa fundamental de la osteoporosis en pacientes con síndrome de Turner ${ }^{6}$. Como en otros casos de talla baja, se ha preconizado la utilización de hormona de crecimiento en pacientes con síndrome de Turner, con resultados modestos en cuanto al pronóstico de talla; no obstante, se ha apreciado que las pacientes tratadas precozmente (antes de la pubertad) con hormona de crecimiento consiguen normalizar su densidad mineral ósea $^{7}$, permaneciendo la controversia sobre el momento idóneo de iniciar el tratamiento sustitutivo estrogénico, teniendo en cuenta su impacto sobre la talla final, pico de edad ósea y desarrollo psicológico de la paciente.

El síndrome de Klinefelter es la alteración más frecuentemente descrita, entre las causas de hipogonadismo hipergonadotrópico del varón. Su incidencia es de 1 cada 300 a 1.000 varones nacidos. La alteración cromosómica más frecuentemente asociada al síndrome es la trisomía del par de cromosomas sexuales, de tipo 47XXY, con polisomías $\mathrm{X}$ ó $\mathrm{Y}$, y diversos mosaicismos descritos. Antes de la pubertad estos pacientes pueden presentar: criptorquidia, micropene, hipospadias, retraso mental, alteraciones del aprendizaje y de conducta, etc. Suelen iniciar la pubertad espontáneamente, retrasada, con grados variables de hipogonadismo posterior. En estos pacientes se ha descrito la presencia de osteoporosis, con incremento en los marcadores de resorción ósea y disminución de los marcadores de formación, con una clara relación entre densidad mineral ósea y niveles de testosterona, lo que sugiere que el hipogonadismo es la causa de la osteoporosis, más que el defecto genético ${ }^{8}$. 
Además de los problemas orgánicos comentados, las alteraciones funcionales del eje gonadal, también pueden acompañarse de una importante repercusión ósea, como sucede en los cuadros descritos en mujeres que realizan una actividad deportiva intensa. La actividad deportiva tiene claros efectos saludables, tanto en varones como en mujeres, reduce la adiposidad, mejora la adaptación cardiovascular, etc.; sin embargo, puede tener su repercusión negativa, en mujeres, afectando tanto a la función reproductiva (inducción de amenorrea primaria o secundaria) como al sistema esquelético (osteopenia, escoliosis y fracturas de estrés).

La relación entre el ejercicio y la masa esquelética es compleja, influyendo además del tipo e intensidad del ejercicio, el área esquelética involucrada, el peso corporal, la dieta, etc. Las atletas que realizan un ejercicio intenso, en actividades que además exigen un control del peso corporal, suelen desarrollar cuadros de amenorrea (primaria antes de la pubertad o secundaria si se ha iniciado la periodicidad menstrual), asociados a disminución de la densidad mineral ósea. En estos casos se ha demostrado la existencia de una disfunción hipotalámica, con disminución de los pulsos de liberación de la hormona liberadora de gonadotropinas $(\mathrm{GnRH})$, que provoca la supresión de la liberación de las hormonas foliculoestimulante (FSH) y luteoestimulante (LH), supresión relativamente más intensa de esta última, manteniendo un patrón secretor típico de la etapa prepuberal, con disminución de la secreción de estrógenos por la gónada. No se ha conseguido identificar el sustrato que puede condicionar este tipo de respuesta hipotalámica, si bien se han implicado el estímulo en la producción de beta-endorfinas por el ejercicio, así como el incremento de actividad del eje hipotálamo-hipofiso-adrenal. No es infrecuente que las atletas con amenorrea desarrollen un trastorno de la conducta alimentaria, con bajo peso. En estos casos se ha demostrado un descenso en los niveles plasmáticos de leptina, hormona segregada por los adipocitos, que podría actuar sobre el "sensor" de la situación energética del organismo, limitando la actividad reproductiva, si bien el papel de la leptina sobre las gonadotropinas todavía está muy debatida ${ }^{9}$.

El síndrome se caracteriza por el trastorno de la conducta alimentaria, amenorrea y pérdida de masa ósea, se denomina con frecuencia "la tríada de la atleta femenina"; pacientes en las que se ha descrito una densidad mineral ósea un 14\% inferior a las mujeres atletas con periodicidad menstrual normal ${ }^{10}$. Las reducciones de masa ósea son significativas en pacientes que han perdido el $50 \%$ de sus ciclos menstruales antes de la edad de 20 años, con el descenso más intenso en columna lumbar, caracterizada por poseer abundante hueso esponjoso, metabólicamente activo.

Todavía no está definitivamente aclarado cuál es el tratamiento más idóneo de estas situaciones; se recomienda disminuir la actividad deportiva e incrementar la dieta, con el objetivo de recuperar el peso corporal. Si no fuera factible y la amenorrea se prolonga más de 6 meses, sería necesario valorar la instauración de tratamiento con anticonceptivos orales, y en mujeres prepuberales, junto con la recuperación del peso, suplementos de calcio oral de unos $1.250 \mathrm{mg} /$ día $^{11}$.

Una situación funcional parecida, con peculiaridades, presentan las pacientes con un trastorno de la conducta alimentaria. Las pacientes con anorexia nerviosa tienen un riesgo aumentado de osteopenia y osteoporosis por la asociación de problemas hormonales: hipoestronismo, hiperparatiroidismo secundario por déficit de vitamina D y calcio, hipercortisolismo y descenso de los niveles del mediador de la hormona de crecimiento (IGF-1), junto a la malnutrición ${ }^{10}$. Si el trastorno aparece antes de haber completado el pico de masa ósea, durante la adolescencia, la disminución de masa ósea será más intensa. Cuando se produce la recuperación, definida como la recuperación del peso y reinicio de la periodicidad menstrual, mejora la mineralización ósea, persistiendo la controversia sobre si esta recuperación es total o parcial, con trabajos recientes que demuestran que a largo plazo la recuperación es parcial $^{12}$. Este mismo grupo encontró que la mayor pér- 
dida de masa ósea se localiza en el fémur, en lugar de la columna, en contraste con estudios previos; sin ninguna correlación entre la duración del período de anorexia o edad de aparición de la enfermedad, con la densidad mineral ósea.

El hipogonadismo hipogonadotropo también parece ser el origen fundamental de otros casos de osteoporosis, asociados a diversas patologías hipofisarias, tal y como se aprecia en pacientes con hiperprolactinemia. En este grupo la densidad mineral ósea es superior en aquellas pacientes con oligomenorrea o periodicidad menstrual normal, que en las que se mantienen amenorreicas ${ }^{10}$; demostrándose una clara mejoría en la mineralización en las pacientes tratadas con agonistas dopaminérgicos, que recuperan la periodicidad menstrual normal ${ }^{13}$; no obstante en algunas series la recuperación no supera el $56 \%$ de las pacientes, quizá por la influencia de otros factores asociados: así se ha descrito un aumento de secreción de péptido relacionado con parathormona (PTHrP) en pacientes con hiperprolactinemia, con correlación negativa entre las concentraciones séricas de PTHrP y densidad mineral ósea, en pacientes con hiperprolactinemia $^{14}$.

En la última década se ha puesto de relevancia el importante papel que juegan los estrógenos en el metabolismo óseo del varón, con la evidencia experimental de que, en ratones, tanto la orquiectomía como la utilización de inhibidores de la aromatasa, que impiden la aromatización de testosterona a estradiol, producen efectos similares sobre la densidad mineral y el recambio óseo ${ }^{15}$. Estos hallazgos se han confirmado en pacientes con alteraciones, tanto en el receptor de estrógenos, como en la actividad aromatasa. Smith describió el caso de un varón de 28 años, portador de una mutación homocigota del gen del receptor de estrógenos, que presentaba un hábito eunucoide, epífisis todavía no cerradas, y osteopenia grave, a pesar de mantener unas concentraciones séricas normales de testosterona y niveles elevados de estradiol ${ }^{16}$. Carani $^{17}$ y Bilezikian ${ }^{18}$ publicaron un caso, cada uno, de varones adultos jóvenes con null-mutaciones del gen de la enzima P-450 aromatasa. Ambos presentaban unos niveles indetectables de estrógenos, con elevación de testosterona, epífisis abiertas y osteopenia. En ambos casos el tratamiento con estrógenos se siguió de la fusión de las epífisis y aumento de la densidad mineral ósea. De estos casos podemos deducir que tanto la falta de respuesta como las alteraciones de la síntesis de estrógenos, en varones, conllevan la aparición de osteopenia. También en estudios poblacionales se ha demostrado que los niveles bajos de estradiol, en varones, se asocian con un elevado recambio óseo y disminución de su densidad mineral ${ }^{19}$. De los estudios experimentales se puede deducir que los estrógenos controlan el 70 \% de la resorción ósea y la testosterona, a través del receptor androgénico, no más del $30 \%$, consistente con el hallazgo de receptores de andrógenos en los osteoclastos, que se integrarían en su sistema regulador de la apoptosis celular. Sin embargo la testosterona puede intervenir más activamente en la diferenciación de osteoblastos, al menos de algunas áreas esqueléticas (formación periostal de hueso $)^{4}$.

\section{OSTEOPOROSIS Y PATOLOGÍA TIROIDEA}

La relación entre masa ósea y situación funcional tiroidea es un tema de la mayor importancia y controvertido en la actualidad. Las hormonas tiroideas son esenciales para el crecimiento y desarrollo durante la infancia así como para el mantenimiento del hueso en la edad adulta. En el niño con hipotiroidismo se aprecia un retraso del crecimiento, con disgenesia epifisaria y retraso de la maduración ósea; y en adultos se prolongan las fases del recambio óseo, con reducción de la actividad osteoblástica e incremento del grosor del hueso cortical. Sin embargo los efectos más acusados de las hormonas tiroideas, sobre el hueso, en la edad adulta, se aprecian en el hipertiroidismo. El hipertiroidismo es una patología muy frecuente, con una prevalencia del $2 \%$ en mujeres y del $0,2 \%$ en varones, apreciándose que a pesar del tratamiento, a largo plazo, la ratio de mortalidad se incrementa en este grupo de población a 2,9, como consecuencia de las secuelas de fracturas 
de cuello de fémur ${ }^{20}$. En una revisión de las consecuencias del hipertiroidismo sobre el hueso, se apreciaba que el $8 \%$ de los pacientes tenían enfermedad ósea sintomática, todas mujeres, la mayoría postmenopaúsicas, de las cuales un $65 \%$ tenían dolor óseo intenso o evidencias de fracturas y hasta el 75 \% habían estado tirotóxicas menos de 1 año ${ }^{21}$.

El mecanismo patogénico de la afectación ósea en el hipertiroidismo se basa en el incremento, tanto del número como de velocidad de recambio, de las unidades de recambio óseo; así están incrementadas las actividades osteoclástica y osteoblástica, con una reducción del tiempo del ciclo de remodelado del $50 \%$ y aumento de la frecuencia de activación de las unidades. Estos cambios conducen a un desacoplamiento entre resorción y formación, con el resultado neto de pérdida de hueso mineralizado, en cantidad variable dependiendo de factores como el sexo, función menstrual, gravedad de la enfermedad tiroidea y sumación de otros factores de riesgo de osteoporosis ${ }^{22}$.

En la actualidad existen pocas dudas sobre el efecto deletéreo del hipertiroidismo sobre el hueso, pero persiste la controversia en dos situaciones que vamos a analizar con más detalle, por su frecuencia y por las implicaciones clínicas que comportan: hipertiroidismo subclínico y tratamiento crónico con hormonas tiroideas.

Las mejoras técnicas han permitido disponer de determinaciones cada vez más sensibles de los niveles de la hormona tirotropa (TSH), que son un fiel indicador de la actividad tisular de la hormona tiroidea (HT), de forma que si sus niveles están suprimidos, aunque todavía los de HT se mantengan dentro de límites normales, podemos hablar de que existe un cierto grado de hipertiroidismo tisular, situación clínica conocida como hipertiroidismo subclínico, con una prevalencia del 1\%, que progresa a hipertiroidismo franco aproximadamente en el 5\% de los casos cada año. Teniendo en cuenta que la pérdida de masa ósea, secundaria al hipertiroidismo, es sólo parcialmente reversible, parece lógico que deberíamos tratar esta situación lo antes posible, si se demuestra que ya está alterando el metabolismo óseo. La controversia persiste, ya que los primeros trabajos demostraban que existía un incremento del recambio óseo, en el hipertiroidismo subclínico; sin embargo, en un trabajo reciente, en el que se examinaban las correlaciones de TSH con la densidad mineral ósea durante un período de seguimiento de 4 a 6 años, no se consiguió demostrar ninguna diferencia entre los grupos con TSH suprimida, normal o elevada $^{23}$; no obstante en otro estudio sí se ha confirmado que las pacientes tratadas para mantener el eutiroidismo conservaban la densidad ósea en columna y cadera, respecto a las no tratadas que seguían con un descenso del $2 \%$ anual $^{24}$. A pesar de la controversia, la tendencia más extendida hoy día, es la de tratar el hipertiroidismo subclínico precozmente, no sólo por sus posibles repercusiones óseas, sino también por los riesgos cardiovasculares que comporta, como por ejemplo la mayor incidencia de arritmias del tipo de la fibrilación auricular ${ }^{25}$.

El tratamiento crónico con hormonas tiroideas y su relación con la osteoporosis es uno de los temas que más trabajos ha suscitado en los últimos años. En este caso debemos centrarnos en dos objetivos terapéuticos claramente diferenciados: el tratamiento supresor con hormonas tiroideas (objetivo suprimir los niveles de TSH, por ejemplo el tratamiento utilizado, tras la cirugía y el radioyodo, en los carcinomas diferenciados de tiroides) y tratamiento sustitutivo (objetivo normalizar los niveles de TSH, por ejemplo el utilizado en el hipotiroidismo primario autoinmune). Con el tratamiento supresor mantenemos al paciente en situación de hipertiroidismo subclínico, apreciándose en la mayoría de trabajos un recambio óseo acelerado. Un metaanálisis reciente que incluía a 1.250 pacientes de 41 estudios, estratificando los pacientes de acuerdo al sexo, estado menopáusico, dosis de HT y lugar anatómico en el que se hizo la densitometría, y excluyendo a los que habían padecido hipertiroidismo previo, concluyó que el tratamiento supresor provocaba una pérdida significativa de masa ósea en columna lumbar y caderas, sólo en mujeres postmenopaúsicas, con un efecto más acusado en 
el hueso cortical. La pérdida era menor de 1 desviación estándar de la media: $7 \%$ en columna lumbar, $5 \%$ en cuello del fémur, $9 \%$ en el triángulo de Ward y $7 \%$ en la porción distal del radio ${ }^{26}$. Todavía estos resultados deben tomarse con cautela y deberían confirmarse con estudios controlados, con estudio tanto de la densidad ósea como de la incidencia de fracturas.

En el caso del tratamiento sustitutivo con HT no se ha demostrado ningún efecto deletéreo sobre el hueso ${ }^{27}$, así que es necesario monitorizar correctamente los niveles de TSH durante el tratamiento a largo plazo para limitar la supresión de TSH sólo a aquellos casos en que sea imprescindible (carcinomas foliculares tiroideos de alto riesgo) y decidir el riesgo-beneficio del tratamiento de la enfermedad nodular tiroidea con dosis supresoras de l-T4.

\section{HIPERPARATIROIDISMO PRIMARIO Y SECUNDARIO}

El hiperparatiroidismo primario es una enfermedad cuyo diagnóstico se ha incrementado dramáticamente en los últimos años, desde que se introdujo la determinación sistemática de la calcemia en los autoanalizadores de canales múltiples, lo que ha comportado que la mayor frecuencia de casos de hiperparatiroidismo primario sean hallazgos casuales en pacientes asintomáticos. La mayor prevalencia se encuentra en grupos de pacientes de edad media, 50 a 60 años, si bien puede diagnosticarse en todas las edades, con una relación mujer/varón de $3 / 1^{28}$. El diagnóstico precoz, en fases asintomáticas, ha comportado que las formas graves de afectación ósea (osteitis fibrosa-quística) sea una rareza clínica, sin embargo sí se aprecia una franca desmineralización en áreas de hueso cortical, si bien en algún grupo de pacientes puede haber una desmineralización preferente de columna ${ }^{29}$. Existe una importante controversia sobre el riesgo real de fracturas en los pacientes con hiperparatiroidismo, si bien existen trabajos claros en los que se demuestra un incremento de riesgo de fractura en antebrazos, vertebras y caderas, tan precoz como 10 años antes de llevar a cabo la paratiroidectomía, normalizándose 1 año después de la intervención ${ }^{30}$.

Dado el alto porcentaje de casos asintomáticos y la necesidad de cirugía para la curación del hiperparatiroidismo, persiste una amplia polémica sobre las indicaciones de la intervención en estos casos, con las siguientes conclusiones de un encuentro reciente, en el que se han analizado los riesgos de la actitud expectante frente a la cirugía $^{31}$, preconizando la intervención quirúrgica en cualquiera de los siguientes casos:

1- Niveles de calcemia superiores en 1 $\mathrm{mg} / \mathrm{dL}$ al límite superior de la normalidad del laboratorio de referencia

2- Calciuria superior a $400 \mathrm{mg}$ en 24 horas.

3- Aclaramiento de creatinina reducido por lo menos en un $30 \%$.

4 - T-score $\leq 2,5$ en cualquier localización del estudio densitométrico.

5- Edad menor de 50 años.

En pacientes ancianos, algunas de las patologías digestivas asociadas con malabsorción y en la insuficiencia renal, se aprecia un déficit de vitamina $\mathrm{D}$ y/o de absorción de calcio intestinal. En casos severos de déficit de vitamina $\mathrm{D}$, la lesión histológica ósea más característica es la osteomalacia; sin embargo en los casos con déficit más larvados, como suelen verse en pacientes ancianos, el déficit comporta el incremento compensador de $\mathrm{PTH}$, con el desarrollo de hiperparatiroidismo secundario, que conlleva la pérdida de masa ósea, acelerando la pérdida que podríamos considerar fisiológica y aproximando al paciente al umbral de fractura ${ }^{32}$.

\section{OTRAS ENDOCRINOPATÍAS}

La involucración del tejido óseo en otras patologías endocrinas está más discutido. Así en la diabetes mellitus tipo 1 se han descrito algunas alteraciones, que podían provocar disminución mineral ósea: aumento de la calciuria por diuresis osmótica, con hiperparatiroidismo secundario asociado, y déficit de insulina e IGF1 , factores importantes para la formación y crecimiento óseos. En estos pacientes, la media de reducción es aproximadamente 
del $8 \%$ en la densidad mineral del esqueleto apendicular'.

Si en el caso anterior puede involucrarse el déficit de IGF-1, el caso inverso puede decirse de la acromegalia, patología en la que se aprecia una elevación de la hormona de crecimiento y su mediador (IGF-1), y en la que se han descrito también casos con disminución de la densidad mineral ósea, no obstante parece que sólo en aquellos en que el tumor hipofisario conlleva la existencia de un hipogonadismo hipogonadotropo ${ }^{2}$.

\section{EVALUACIÓN DIAGNÓSTICA DE CAUSAS DE OSTEOPOROSIS SECUNDARIA}

En cualquier paciente que se diagnostica de osteoporosis pueden encontrarse causas secundarias que hayan contribuido a la progresión del cuadro clínico; así en aquellos que presenten una pérdida de masa ósea, superior a la esperada, de acuerdo a su edad, sexo, etnia y estatus gonadal debería plantarse un despistaje de causas de osteoporosis secundaria.

Todos los varones, mujeres premenopáusicas o perimenopaúsicas que hayan sufrido fracturas no traumáticas, o que su densidad mineral ósea sea 1 desviación estándar inferior a los sujetos control de su edad y sexo, requerirían un estudio más intensivo ${ }^{2}$.

La Sociedad Americana de Endocrinólogos Clínicos recomienda el siguiente estudio para identificar causas de osteoporosis secundaria ${ }^{33}$ :

1- Estudio basal: hemograma completo, bioquímica sérica (calcio, fosforo, proteínas totales, albúmina, enzimas hepáticas, fosfatasa alcalina, creatinina y electrolitos) y calcio en orina de 24 horas.

2- Estudio dirigido según los datos clínicos obtenidos de la historia y exploración física: TSH sérica, PTH sérica, concentración sérica de 25 hidroxi-vitamina D, cortisol libre en orina (con estudios dinámicos según resultados), estudio del metabolismo ácido-base, marcadores bioquímicos del recambio óseo, triptasa sérica o $\mathrm{N}$-metilhistamina en orina para descartar mastocitosis, electroforesis sérica o urina- ria, aspirado de médula ósea, biopsia de hueso iliaco (sólo si es grave sin causa aparente, no hay respuesta al tratamiento, sospecha de osteomalacia o mastocitosis).

La lista previa no es exhaustiva y posiblemente se irá incrementando en los próximos años. Al menos en la lista se acusa la falta de algún estudio dirigido a descartar la presencia de una enfermedad celiaca del adulto, que en muchos casos puede ser clínicamente asintomática y que algunas series se ha diagnosticado hasta en el $12 \%$ de pacientes con osteoporosis postmenopaúsicas ${ }^{3}$. El diagnóstico de sospecha puede realizarse con bastante sencillez, mediante la determinación de anticuerpos antigliadina, antiendomisio y antitransglutaminasa, si bien el diagnóstico de certeza debe hacerse mediante la biopsia endoscópica de intestino delgado.

El estudio sistemático de causas de osteoporosis secundaria en un hospital terciario, incluyendo a 664 pacientes, con exclusión de aquellas que hubieran recibido alguna medicación que pudiera alterar el metabolismo óseo o con antecedentes de una clara patología que justificase los hallazgos, cumpliendo criterio de inclusión 173 mujeres, arrojó los siguientes datos: el $32 \%$ tenía alguna patología, no diagnosticada, del metabolismo mineral, que en el $85 \%$ de los casos podían diagnosticarse con la determinación del calcio sérico y urinario, niveles de PTH sérica y TSH sérica en aquellas pacientes en tratamiento con hormonas tiroideas, con un coste de $75 \$ /$ paciente $^{34}$. La conclusión evidente es que la existencia de alteraciones, previamente no diagnosticadas, en pacientes con osteoporosis postmenopáusica y sin otros problemas de salud, es frecuente, pudiendo identificarse la mayoría de ellas con una estrategia sencilla y barata.

\section{BIBLIOGRAFÍA}

1. Osteoporosis prevention, diagnosis, and therapy. NIH Consensus Stratement 2000; 17 : 1-45. Accesed at http://odp.od.nih.gov/consensus/cons/111/1 11_statement.htm.

2. Stein E, Shane E. Secondary osteoporosis. Endocrinol Metab Clin N Am 2003; 32: 115134. 
3. HARPER KD, Weber TJ. Secondary osteoporosis: diagnostic considerations. Endocrinol Metab Clin N Am 1998; 27: 325348.

4. RigGs BL, Sundeep K, Melton III J. Sex steroids and the construction and conservation of the adult skeleton. Endocr Rev 2002; 23: 279302.

5. LIPPE B. Turner syndrome . Endocrinol Metab Clin N Am 1991; 20: 121-152.

6. Davies MC, GluleKLI B, JaKoBS HS Osteoporosis in Turner's syndrome and other forms of primary amenorrhea. Clin Endocrinol (Oxford) 1995; 43: 741-746.

7. NEely EK, Marcus R, Rosenfeld RG, Bachrach LK. Turner syndrome adolescents receiving growth hormone are not osteopenic. J Clin Endocrinol Metab 1993; 76: 861-866.

8. Horowitz M, Wishart JM, O`LOUGHLIN PD. Osteoporosis and Klinefelter syndrome. Clin Endocrinol 1992; 36: 113-118.

9. Warren MP, Voussoughian F, GeER EB, Hyle EP, ADBERG CI, RAmos RH. Functional hypotalamic amenorrhea: hypoleptinemia and disordered eating. J Clin Endocrinol Metab 1999; 84: 873-877.

10. MilLer KK, KliBansky A. Amenorrheic bone loss. J Clin Endocrinol Metab 1999; 84: 17751783 .

11. WarRen MP, Shanta S. Anorexia, bulimia, and the endocrinology of execise. In Wass J.A.H., and Shalet S.M. editors. Oxford textbook of Endocrinology 2002: 267-271.

12. Hartman D, Crisp A, Rooney B, Rackow C, ATKINSON R, PATEL S. Bone density of women who have recovered from anorexia nervosa. Int J Eat Disord 2000; 28: 107-112.

13. SANFILIPPO JS. Implications of not treating hyperprolactinemia. J Reprod Med 1999; 44: 1111-1115.

14. Stiegler C, Leb G, Kleinart R, Warnkross H, RAMSCHAK-SCHWARZER S, LIPP R, et al. Plasma levels of parathyroid hormone-related peptide are elevated in hyperprolactinemia and are correlated to bone density status. J Bone Miner Res 1995; 10: 751-759.

15. VANDERsChueren D, van Herck E, DE Coster R, BOUILLON R. Aromatization of androgens is important for eskeletal maintenance of aged male rats. Calcif Tissue Int 1996; 59: 179-183.

16. SMith EP, Boyd J, FRANK GR, TAKahashi H, COHEN RM, SPECKER B et al. Estrogen resistance caused by a mutation in the estrogen-receptor gene in man. $\mathrm{N}$ Eng J Med 1994; 331: 1056-1061.
17. Carani C, Qin K, Simoni M, Faustini-Faustini M, SERPENTE S, BOYD J et al, Simpson ER. Effect of testosterone and estradiol in a man with aromatase deficiency. N Eng J Med 1997; 337: 91-95.

18. Bilezikian JP, Morishima A, Bell J, Grumbach MM. Increased bone mass as a result of estrogen therapy in a man with aromatase deficiency. N Eng J Med 1998; 339: 599-603.

19. Szulc P, Munoz F, Claustrat B, Garnero P, Marchand F, Duboeuf $F$ et al. Bioavailable estradiol may be an important determinant of osteoporosis in men: The MINOS study. J Clin Endocrinol Metab 2001; 86: 192-199.

20. Franklin JA, MaIsonNeuve P, ShepPaRd MC, BETTERIDGe J, Boyle P. Mortality after the treatment of hypertyroidism with radioactive iodine. N Eng J Med 1998; 338: 712-718.

21. Ross DS. Bone disease in hyperthyroidism. En: Avioli LV. Krane SM. Eds. Metabolic Bone Disease. San Diego: Academic Press 1998: 531-544.

22. MoseKilde I, ERIKSEn EF, Charles P. Effects of thyroid hormones on bone and mineral metabolism. Endocrinol Metab Clin N Am 1990; 19: 35-63.

23. Bauer DC, Nevitt MC, Ettinger B, Stone K. Low thyrotropin levels are not associated with bone loss in older women: a prospective study. J Clin Endocrinol Metab 1997; 82: 2931-2936.

24. Faber J, Jensen IW, Petersen I, Nygaar B, Hegedus I, Siersbaek Nielsen K. Normalization of serum thyrotropin by means of radioiodine treatment in subclinical hyperthyroidism: effect on bone loss in postmenopausal women. Clin Endocrinol 1998; 48: 285-290.

25. SaWin CT, Geller A, Wolf PA, Belanger AJ, BAKER E, BACHARACH $P$ et al. Low serum thyrotropin concentration as a risk factor for atrial fibrillation in older persons. N Eng J Med 1994; 331: 1249-1252.

26. Uzzan B. Campos J, Cucherat M, Nony P, Boissel JP, PERRET GY. Effects on bone mass of long term treatment with thyroid hormones: a meta-analysis. J Clin Endocrinol Metab 1996; 81: 4278-4289.

27. Hanna FW, Pettit RJ, Ammari F, Evans WD, SANDERMAN D, LaZARus JH. Effect of replacement doses of thyroxine on bone mineral density. Clin Endocrinol 1998; 48: 229-234.

28. Heath H, Hodgson SF, Kennedy MA. Primary hyperparathyroidism: incidence, morbidity, 
and economic impact in a community. N Eng J Med 1980; 302: 189-193.

29. Parisien M, Silverberg SJ, Shane E, Dempster DW, BILEZIKIAN JP. Bone disease in primary hyperprathyroidism. Endocrinol Metab Clin N Am 1990; 19: 19-34.

30. Vestergatad P, Mollerup CL, Frokjaer VG, Christiansen R, Blichert-Toft $\mathrm{H}$, MoseKILDE I. Cohort study of risk of fracture before and after surgery for primary hyperparathyroidism. Br Med J 2000; 321: 598-602.

31. BILEZIKIAN JP, PotTS JT, FUlEIHAN GEH, Kleerekoper M, NeER R, PeAcock $M$ et al. Summary Statement from a Workshop on Asymptomatic Primary Hyperparathyroidism: A Perspective for the $21^{\text {st }}$ Century. J Clin Endocrinol Metab 2002; 87: 5353-5361

32. LIPS P. Vitamin D Deficiency and Secondary Hyperparathyroidism in the Elderly: Consequences for bone loss and Fractures and Therapeutic Implications. Endocr Rev 2001; 22: 477-501.

33. American Association of Clinical Endocrinologist 2001 medical guidelines for clinical practice for the prevention and management of postmenopausal osteoporosis. Endocr Pract 2001; 7: 293-312.

34. Tannenbaum C, Clark J, Schwartzman K, WALLENSTEIN S, LAPINSKI R, MEIER D et al. Yield of laboratory testing to identify secondary contributors to osteoporosis in otherwise healthy women. J Clin Endocrinol Metab 2002; 87: 4431-4437. 